\title{
A NEW APPROACH TO THE ANALYSIS OF A DISCRETE ROUND-ROBIN QUEUE
}

Abstract. We identify the regions of parameters of the arriving stream in which the ergodic, critical, or supercritical properties of the branching chain are established.

1. Introduction. In this paper we study a processor working under the round-robin (RR) algorithm which is discussed, for example, in $[2,4,9,10]$.

In this section the standard model of a processor operating under the $\mathrm{RR}$ discipline will be described. We give a concise presentation of most important notions and results from the literature. In Subsections 1.3 and 1.4 we present our main notations concerning the RR processor and give a short description of our results.

1.1. Description of the standard discrete time model of a round-robin processor. The RR service works in such a way that out of a queue of signals awaiting in the buffer (which, it is assumed, has infinite capacity) the one found at the very head of the queue is selected for service. That signal receives one service time slice equal to $q$. If this exhausts the signal's required service time, the signal exits the system; otherwise, the signal positions itself at the tail of the queue with a service time diminished by $q$. Signals enter the system from the outside at random moments and have random required service times. The random variables describing these two values can have discrete distribution as in the works of Schassberger, or continuous distribution as proposed by Grishechkin, for example. A mathematical model describing the status of the system is an appropriate Markov chain.

1.2. A brief overview of earlier papers on $R R$. In [1] Daduna analyzes a discrete-time round-robin queue with the last-in-first-served rule: a newly

2000 Mathematics Subject Classification: 60G35, 60K25, 90B18, 90B22.

Key words and phrases: ergodicity, linear speed of growth property, multitype branching process, round-robin processor, supercriticality. 
arriving signal receives a quantum of service immediately and only thereafter joins the tail of the queue. Signals are of different types, the set $R$ of possible types $r \in R$ being at most countably infinite. At the end of each quantum at most one arrival to the queue may occur, the sequence of arrivals being Bernoulli with probability $\lambda \in(0,1)$ for an arrival. Each arrival presents a request for service. The probability for a request of $k$ quanta is $\pi_{r}(k), k \leq 1$, for a type- $r$ signal. The model is studied in terms of a discrete-time Markov chain with state space $\{e\} \cup\left\{\left(r_{n}, \ldots, r_{1}\right): n=1,2, \ldots, r_{i} \in R, 1 \leq i \leq n\right\}$ where $R$ is finite or countably infinite. The element $\left(r_{n}, \ldots, r_{1}\right)$ represents the state of the queue of signals, the signal at the head of the queue being of type $r_{1}, \ldots$, at the tail of the queue of type $r_{n}$. The element $e$ represents the empty queue. Daduna computes steady-state probabilities and the mean sojourn time.

In [10] Schassberger analyzes a model with one type signals where queue statuses at times $0, q, \ldots$ are defined as either $e$ (for empty) or else $\left(k_{n}, \ldots, k_{1}\right)$ where $k_{i} \in\{1,2, \ldots\}$ and $k_{1} q$ is the residual demand of the signal at the head of the queue, $\ldots, k_{n} q$ is the residual demand of the signal at the tail of the queue. Schassberger examines the sojourn time of the signal with required service time $k q$, where $k \in \mathbb{N}$, and utilizes the generating function of the random variable that describes the sojourn time. Moreover, he points out that as $q \rightarrow 0$ (and with suitably selected parameters of the input stream), the distribution of the sojourn time tends to the distribution of the sojourn time of a signal in an appropriate system with a processor working under the processor sharing (PS) discipline.

In [4], Grishechkin analyzes a processor model with continuous time and RR rules, where the input stream is a Poisson stream and the required service time has exponential distribution. He shows that the dynamics of the Markov chain describing the queue status is tantamount to the dynamics of an appropriate branching process with immigration. His main focus is also on the sojourn time in the system of a distinguished signal which has a given required service time. Application of the branching processes theory allows him to prove limit theorems, for example, when the number of signals present in the system at the moment of appearance of the distinguished signal tends to $\infty$, or when the distinguished signal's required service time tends to $\infty$. Grishechkin's analysis is quite difficult to follow.

In this paper we propose a more lucid model and a more intuitive application of branching processes. To analyze the dynamics of a branching process, the ideas put forward in $[5,6,8]$ will be used. Parameter areas will be indicated in which the Markov chain describing the system status exhibits ergodic, critical, or supercritical behavior; this was never considered in the above-mentioned works. The manner of describing the system status proposed in this paper and the results arrived at also allow estimating the 
sojourn time and the departure process in each of the three indicated areas. However, detailed calculations have been omitted as they can easily be deduced from the results obtained.

1.3. $R R$ processor with apparent signals and the appropriated multitype branching chain. The processor we consider has an unbounded waiting room in which the currently served signals are ordered in a queue. The real signals arrive one by one from the outside at successive times $0<T_{1}<T_{2}<\cdots$, where

$$
P\left(\left\{T_{1}=l q\right\}\right)=P\left(\left\{T_{i+1}-T_{i}=l q\right\}\right)=(1-\gamma)^{l-1} \gamma
$$

for $i, l \in \mathbb{N} ; \gamma \in(0,1)$ and $q \in(0, \infty)$ are parameters.

The signal arriving at time $T_{i}$ presents a demand for service time of size $S_{i}$, where

$$
\begin{gathered}
P\left(\left\{S_{i}=l q\right\}\right)=\pi_{l}, \quad l=1, \ldots, k+1, i \geq 1, \\
\sum_{l=1}^{k+1} \pi_{l}=1 .
\end{gathered}
$$

We assume that the random variables

$$
T_{1}, S_{1}, T_{2}-T_{1}, S_{2}, T_{3}-T_{2}, S_{3}, \ldots \text { are independent }
$$

and are viewed as being defined on a suitably chosen probability space with probability measure $P$.

We can associate to the arriving signal stream the Bernoulli chain $\left\{b_{n}\right.$ : $n \in \mathbb{N}\}\left(b_{1}, b_{2}, \ldots\right.$ are independent, $\left.P\left(\left\{b_{n}=1\right\}\right)=\gamma, P\left(\left\{b_{n}=0\right\}\right)=1-\gamma\right)$ in the following way: if $b_{n}=1$, then the signal arrives at time $n q$. Apart from real signals we will introduce apparent ones at times $0<V_{1}<V_{2}<\cdots$ with required service time $q$. The times $V_{1}, V_{2}, \ldots$ are random variables and now we are going to describe their practical meaning. The formal definition will be given later.

In the queue there are real signals whose residual request is greater than zero. In the time period $[0, q)$ an apparent signal is served. Next, if $T_{1}=q$, then in the time period $[q, 2 q)$ the first real signal is served, otherwise $V_{1}=q$ and the second apparent signal is served in $[q, 2 q)$. In the next time periods $[k q,(k+1) q), k \in \mathbb{N}$, the consecutive signal (real or apparent) from the head of the queue (as at time $k q$ ) takes its one service quantum. One service quantum is the processor's work during a time period of length $q$. So suppose the signal finishes taking its quantum of service at time $(k+1) q$. Then it goes to the end of the queue if its remaining request is greater than zero, otherwise it departs the system. If at time $(k+1) q$ a new real signal arrives from the outside it takes its place at the head of the queue and the signals presently waiting in the queue are moved one place backward. At time $(k+1) q$ the 
signal which is actually at the head of the queue goes to the processor and it takes its service quantum in the interval $[(k+1) q,(k+2) q)$.

Apparent signals are moved to the head of the queue only at times $V_{j}$, $j \in \mathbb{N}^{*}$ where $\mathbb{N}^{*}:=\{0\} \cup \mathbb{N}$, so that in the time interval $\left[V_{n}, V_{n+1}\right)$ an apparent signal as well as any other real signal from the queue will receive exactly one quantum of service. The interval $\left[V_{n}, V_{n+1}\right), n \in \mathbb{N}^{*}$, will be called the $(n+1)$ th period.

Let $Z_{j}(n), j \in\{1, \ldots, k\}$, represent the number of signals in the queue with the residual service time at time $V_{n}$ equal to $j q$. Set $\bar{Z}(n):=\left(Z_{1}(n), \ldots\right.$, $\left.Z_{k}(n)\right)$. Then $Z(n)=\sum_{j=1}^{k} Z_{j}(n)$ is the length of the queue at time $V_{n}$.

Now we give the formal recurrent definition for the sequence $\left\{V_{n}, \bar{Z}(n)\right.$ : $n \in \mathbb{N}\}$. Let $\left\{b_{l}^{(m, n)}: l \in \mathbb{N}\right\}, m, n \in \mathbb{N}^{*}$, be a sequence of independent Bernoulli chains defined on the same probability space as $\left\{T_{j}, S_{j}: j \in \mathbb{N}\right\}$. This sequence has the same distribution as the sequence $\left\{b_{n}: n \in \mathbb{N}\right\}$. For $m, n \in \mathbb{N}^{*}$ we define

$$
B_{n}^{(m)}:= \begin{cases}0 & \text { if } b_{1}^{(m, n)}=0, \\ \inf \left\{l: b_{1}^{(m, n)}=\cdots=b_{l}^{(m, n)}=1, b_{l+1}^{(m, n)}=0\right\} & \text { if } b_{1}^{(m, n)}=1 .\end{cases}
$$

Notice that $P\left(\left\{B_{n}^{(m)}<\infty\right\}\right)=1$.

DeFinition 1.1. We define

$$
V_{1}:=q\left(1+B_{0}^{(0)}\right)
$$

and for $l=1, \ldots, k$,

$$
Z_{l}(1):=\left\{\begin{array}{l}
0 \quad \text { if } B_{0}^{(0)}=0, l \in\{2, \ldots, k\}, \\
1 \quad \text { if } B_{0}^{(0)}=0, l=1, \\
1+B_{0}^{(0)}-\#\left\{j \in\left\{1, \ldots, B_{0}^{(0)}\right\}: S_{j} \neq(l+1) q\right\} \quad \text { if } B_{0}^{(0)} \geq 1 .
\end{array}\right.
$$

Having $\left(V_{1}, \bar{Z}(1)\right), \ldots,\left(V_{n}, \bar{Z}(n)\right)$ we define

$$
Z(n)=\sum_{l=1}^{k} Z_{l}(n), \quad V_{n+1}=V_{n}+q\left(1+\sum_{j=1}^{Z(n)} B_{n}^{(j)}\right) .
$$

Now in the case when $\sum_{j=1}^{Z(n)} B_{n}^{(j)}=0$ we put $Z_{1}(n+1):=1+Z_{2}(n)$, $Z_{l}(n+1):=Z_{l+1}(n)$ when $l \in\{2, \ldots, k-1\}$ and $Z_{k}(n+1)=0$.

When $\sum_{j=1}^{Z(n)} B_{n}^{(j)} \geq 1$ we first define

$$
A_{(n)}^{(i)}:=B_{0}^{(0)}+\sum_{l=1}^{n-1}\left(\sum_{j=1}^{Z(l)} B_{l}^{(j)}\right)+I(i>1) \sum_{j=1}^{i-1} B_{n}^{(j)},
$$

where $I(i>1)=0$ when $i=0$ or $i=1$, and $I(i>1)=1$ when $i>1$. Then 
we define for $l=1, \ldots, k$,

$$
\begin{aligned}
Z_{l}(n+1)= & (1-I(l>1))+Z_{l+1}(n) \\
& +\sum_{i=1}^{Z(n)}\left(B_{n}^{(i)}-\#\left\{j \in\left\{1, \ldots, B_{n}^{(i)}\right\}: S_{A_{(n)}^{(i)}+j} \neq(l+1) q\right\}\right) .
\end{aligned}
$$

For $l \in\{1, \ldots, Z(n-1)\}, n \in \mathbb{N}$, let $T(l ; n)$ denote the time when the $l$ th signal from the queue (i.e. $l$ th at time $\left.V_{n-1}\right)$ finishes taking its one quantum of service. Then $\xi_{n+1, l}:=q^{-1}(T(l+1 ; n)-q-T(l ; n))$ equals the number of new real signals which arrived from the outside one by one beginning from time $T(l ; n)$.

For $m \in \mathbb{N}^{*}$ we have

$$
\begin{array}{r}
P\left(\left\{\xi_{n+1, l}=m\right\}\right)=P\left(\left\{b_{j}=1, j \in\left\{q^{-1} T(l ; n), \ldots, q^{-1} T(l ; n)+m-1\right\}\right.\right. \\
\left.\left.b_{j_{1}}=0, j_{1}:=m+q^{-1} T(l ; n)\right\}\right)=(1-\gamma) \gamma^{m}
\end{array}
$$

Let $\xi_{n, l}^{(j)} \in\{0\} \cup \mathbb{N}$ denote the number of signals in the set of all $\xi_{n, l}$ signals with the required service time $j q, j \in\{1, \ldots, k+1\}$. Obviously $\sum_{j=1}^{k+1} \xi_{n, l}^{(j)}=\xi_{n, l}$.

According to the assumption (1.1) we can use the Bernoulli scheme to conclude that for each $i \in\{1, \ldots, k+1\}$,

$$
P\left(\left\{\xi_{n, l}^{(i)}=j\right\} \mid\left\{\xi_{n, l}=m\right\}\right)= \begin{cases}\left(\begin{array}{c}
m \\
j
\end{array}\right) \pi_{i}^{j}\left(1-\pi_{i}\right)^{m-j}, & 0 \leq j \leq m, \\
1, & m=j=0 .\end{cases}
$$

Hence if $\xi_{n, l}=m \geq 1$, then $\left(\xi_{n, l}^{(1)}, \ldots, \xi_{n, l}^{(k+1)}\right)$ has multinomial distribution with $k+1$ components:

$$
P\left(\left\{\xi_{n, l}^{(1)}=j_{1}, \ldots, \xi_{n, l}^{(k+1)}=j_{k+1}\right\} \mid\left\{\xi_{n, l}=m\right\}\right)=\overline{\left(\begin{array}{c}
m \\
j
\end{array}\right)} \prod_{i=1}^{k+1} \pi_{i}^{j_{i}}
$$

where

$$
\overline{\left(\begin{array}{c}
m \\
j
\end{array}\right)}:=\frac{m !}{j_{1} ! \cdots j_{k+1} !} .
$$

In view of the assumption (1.1) the random vectors

$$
\left\{\left(\xi_{n, l}^{(1)}, \ldots, \xi_{n, l}^{(k+1)}\right): n \in \mathbb{N}^{*}, l \in\{1, \ldots, Z(n)\}\right\}
$$

are independent.

From the description of the processor's operation we can derive the following recurrence relation: 


$$
\begin{aligned}
Z_{j}(n+1) & =Z_{j+1}(n)+\sum_{l=1}^{Z(n)} \xi_{n+1, l}^{(j+1)}+\delta_{j, 1}, \quad j=1, \ldots, k-1, \\
Z_{k}(n+1) & =\sum_{l=1}^{Z(n)} \xi_{n+1, l}^{(k+1)}
\end{aligned}
$$

and $\bar{Z}(0)=e_{1}:=(1,0, \ldots, 0)$.

So $\bar{Z}(n)$ describes the population of $k$ types of individuals in generation $n \in \mathbb{N}^{*}$. In the next generation the individuals from the previous one change their type from $j$ to $j-1, j \in\{2, \ldots, k\}$, and there appears one new individual of type 1 . The number of individuals in the $n$th generation is $Z(n)$. For each individual $l \in\{1, \ldots, Z(n)\}$ in the $n$th generation, $\xi_{n+1, l}^{(i)}$ denotes the number of its children of type $i$. We showed that whenever $\bar{Z}(0)=(1,0, \ldots, 0)$ then $\bar{Z}(n), n \in \mathbb{N}$, describes the state of the queue in the buffer of the RR processor at the beginning of the $(n+1)$ th period.

Notice that

$$
V_{n+1}-V_{n}=q\left(Z(n+1)+Z_{1}(n)+\sum_{l=1}^{Z(n)} \xi_{n+1, l}^{(1)}+1\right), \quad n \in \mathbb{N}^{*}
$$

is the duration time of the $(n+1)$ th processor operation cycle.

Let $t(m ; n)$ denote the sojourn time for a signal of demand $m q$ arriving during the $n$th period. Then we have the estimate

$$
V_{n+m-1}-V_{n}<t(m ; n)<V_{n+m-1}-V_{n-1} .
$$

Finally, note that the number $D(n+1)$ of real signals departing the system during the $(n+1)$ th period is equal to

$$
D(n+1)=Z_{1}(n)+\sum_{l=1}^{Z(n)} \xi_{n+1, l}^{(1)} .
$$

So we conclude that whenever we find the chain $\bar{Z}(n), n \in \mathbb{N}^{*}$, we obtain information about the sojourn time of signals and about the departure process.

1.4. Brief description of the results. In Section 2 we give a complete Frobenius-Perron analysis for the matrix describing the dynamics of $E(\bar{Z}(n)), n \in \mathbb{N}^{*}$. This will allow us to divide the domain of parameters $D:=\left\{\left(\gamma, \pi_{1}, \ldots, \pi_{k+1}\right): \gamma \in(0,1), \pi_{i} \in(0,1), i=1, \ldots, k+1, \sum_{i=1}^{k+1} \pi_{i}\right.$ $=1\}$ into subdomains $D_{\mathrm{e}}, D_{\mathrm{c}}, D_{\text {sup }}$ where the dynamics of the chain is ergodic, critical and supercritical respectively.

The ergodicity will be proved in Subsection 4.1. We apply the Foster theorem [3]. For the construction of the appropriate Lyapunov function we use ideas from [5]. 
In Subsection 4.2 we identify the subdomain $D_{\mathrm{c}}^{l} \subset D_{\mathrm{c}}$ such that $\bar{Z}(n)$, $n \in \mathbb{N}^{*}$, has a linear speed of explosion with respect to $n$ when the parameters are from $D_{\mathrm{c}}^{l}$. The concepts from $[5,6]$ will be applied.

In Subsection 4.3 we give a result of Kesten-Stigum type when the parameters are from $D_{\text {sup }}$. Here we apply a classical argument from the book [11].

In the relevant literature the question of ergodicity or the critical or supercritical behavior of Markov chain models for RR systems has not been studied. Our notion of the chain $\{\bar{Z}(n): n \in \mathbb{N}\}$ makes it possible to pose such questions and give answers. It is the principal contribution of our paper to the analysis of RR-systems.

2. Basic analysis of the chain $\bar{Z}(n), n \in \mathbb{N}^{*}$. In view of (1.3), (1.4) we find that

$$
\begin{aligned}
P\left(\left\{\xi_{n, l}^{(i)}=j\right\}\right) & =\sum_{m=j}^{\infty} P\left(\left\{\xi_{n, l}^{(i)}=j\right\} \mid\left\{\xi_{n, l}=m\right\}\right) \cdot P\left(\left\{\xi_{n, l}=m\right\}\right) \\
& =(1-\gamma) \sum_{m=j}^{\infty}\left(\begin{array}{c}
m \\
j
\end{array}\right) \pi_{i}^{j}\left(1-\pi_{i}\right)^{m-j} \gamma^{m} \\
& =(1-\gamma)\left(\frac{\pi_{i}}{1-\pi_{i}}\right)^{j} g_{j}\left(\left(1-\pi_{i}\right) \gamma\right),
\end{aligned}
$$

where

$$
g_{j}(s):=\sum_{m=j}^{\infty}\left(\begin{array}{c}
m \\
j
\end{array}\right) s^{m}, \quad 0<s<1 .
$$

For the functions $g_{j}, j \in \mathbb{N}$, we derive

$$
g_{j+1}(s)=\frac{1}{j+1}\left(s g_{j}^{\prime}(s)-j g_{j}(s)\right), \quad j \in \mathbb{N},
$$

and we calculate immediately

$$
g_{1}(s)=\frac{s}{(1-s)^{2}} .
$$

From these we obtain

$$
g_{j}(s)=\frac{s^{j}}{(1-s)^{j+1}}, \quad j \in \mathbb{N} .
$$

Hence

$$
P\left(\left\{\xi_{n, l}^{(i)}=j\right\}\right)=\frac{(1-\gamma)\left(\pi_{i} \gamma\right)^{j}}{\left(1-\gamma\left(1-\pi_{i}\right)\right)^{j+1}}, \quad j \geq 1 .
$$

For $j=0$ we deduce immediately

$$
P\left(\left\{\xi_{n, l}^{(i)}=0\right\}\right)=\frac{1-\gamma}{1-\gamma\left(1-\pi_{i}\right)} .
$$


Next we calculate

$$
\begin{aligned}
m_{j} & :=E\left(\xi_{n, l}^{(j)}\right)=\sum_{m=1}^{\infty} E\left(\xi_{n, l}^{(j)} \mid\left\{\xi_{n, l}=m\right\}\right) \cdot P\left(\left\{\xi_{n, l}=m\right\}\right) \\
& =(1-\gamma) \pi_{j} \sum_{m=1}^{\infty} m \gamma^{m}=\frac{\gamma}{(1-\gamma)} \pi_{j}, \quad j=1, \ldots, k+1,
\end{aligned}
$$

and similarly

$$
c_{j j}:=D^{2}\left(\xi_{n, l}^{(j)}\right)=\frac{\gamma}{1-\gamma} \pi_{j}\left(1-\pi_{j}\right), \quad j=1, \ldots, k+1 .
$$

Because the conditional distribution of the random vector $\left(\xi_{n, l}^{(i)}, \xi_{n, l}^{(j)}\right), i \neq j$, conditioned on the event $\left\{\xi_{n, l}=m\right\}$ is the trinomial distribution with the generating function

$$
g_{m}\left(s_{1}, s_{2}\right)=\left(1-\pi_{i}-\pi_{j}+s_{1} \pi_{i}+s_{2} \pi_{j}\right)^{m},
$$

for $i \neq j$ we have

$$
E\left(\xi_{n, l}^{(i)} \xi_{n, l}^{(j)} \mid\left\{\xi_{n, l}=m\right\}\right)=\left.\frac{\partial^{2}}{\partial s_{1} \partial s_{2}} g_{m}\left(s_{1}, s_{2}\right)\right|_{s_{1}=s_{2}=1}=m(m-1) \pi_{i} \pi_{j} .
$$

From this we obtain

$$
c_{i j}:=\operatorname{cov}\left(\xi_{n, l}^{(i)}, \xi_{n, l}^{(j)}\right)=\frac{\gamma^{2}}{(1-\gamma)^{2}} \pi_{i} \pi_{j}, \quad i \neq j, i, j \in\{1, \ldots, k+1\} .
$$

We define the matrices

$$
\begin{gathered}
\mathcal{L}=\left(\begin{array}{cccccc}
0 & 0 & 0 & 0 & \cdots & 0 \\
1 & 0 & 0 & 0 & \cdots & 0 \\
0 & 1 & 0 & 0 & \cdots & 0 \\
\vdots & \vdots & \ddots & \ddots & \ddots & \vdots \\
0 & 0 & \cdots & 1 & 0 & 0 \\
0 & 0 & \cdots & 0 & 1 & 0
\end{array}\right), \quad \mathcal{K}=\left(\begin{array}{cccc}
m_{2} & \left.m_{3}\right)_{i, j=2}^{k+1} & \cdots & m_{k+1} \\
m_{2} & m_{3} & \cdots & m_{k+1} \\
\vdots & \vdots & & \vdots \\
m_{2} & m_{3} & \cdots & m_{k+1}
\end{array}\right), \\
\\
\mathcal{M}=\mathcal{L}+\mathcal{K} .
\end{gathered}
$$

Notice that all entries of $\mathcal{M}$ are positive.

The recurrence (1.5) can be rewritten in the form

$$
\begin{aligned}
\bar{Z}(n+1) & =\bar{Z}(n) \mathcal{M}+\bar{\zeta}(n+1)+e_{1}, \quad n \geq 0, \\
\bar{Z}(0) & =e_{1},
\end{aligned}
$$


where

$\bar{\zeta}(n):=\left(\zeta_{2}(n), \ldots, \zeta_{k+1}(n)\right), \quad \zeta_{j}(n):=\sum_{l=1}^{Z(n-1)}\left(\xi_{n, l}^{(j)}-m_{j}\right), \quad j=2, \ldots, k+1$.

Iterations of (2.1) yield

$$
\bar{Z}(n+1)=e_{1} \sum_{i=0}^{n+1} \mathcal{M}^{i}+\sum_{i=0}^{n} \bar{\zeta}(n+1-i) \mathcal{M}^{i}, \quad n \geq 0 .
$$

LEMMA 2.1. The matrix $\mathcal{M}$ is irreducible.

We recall from [2] that an $m \times m$ matrix $\mathbf{M}$ is called irreducible if for every pair $(i, j)$ of indices there exists an integer $n=n(i, j)$ such that $m_{i j}^{(n)}>0$, where $\left(m_{i j}^{(n)}\right)_{i, j=1}^{m} \equiv \mathbf{M}^{n}$.

The Perron-Frobenius theorem for irreducible matrices states that the eigenvalue of $\mathcal{M}$ having the largest absolute value is simple and positive. We denote this eigenvalue by $\varrho$. Moreover, if $v$ and $u$ denote left and right eigenvectors of $\mathcal{M}$ corresponding to $\varrho$, then all the coordinates of $v$ and $u$ are positive.

We choose $v, u$ such that $v \cdot u=1$, and $\sum_{j=1}^{k} u_{j}=1$. Define

$$
\lambda:=E\left(T_{1}\right)=\frac{1-\gamma}{\gamma}, \quad r:=\lambda^{-1}(\mu-1), \quad \mu:=E\left(S_{n}\right)=\sum_{j=1}^{k+1} j \pi_{j} .
$$

THEOREM 2.2. If $r \in(0,1)$, then $\varrho<1$; if $r=1$, then $\varrho=1$; and if $r>1$, then $\varrho>1$.

Proof. Let $\left(v_{1}, \ldots, v_{k}\right) \equiv v$. Rewrite the equation $\varrho v=v \mathcal{M}$ in the form

$$
\varrho\left(v_{1}, \ldots, v_{k}\right)=\left(v_{2}, \ldots, v_{k}, 0\right)+\lambda^{-1} V \cdot\left(\pi_{2}, \ldots, \pi_{k+1}\right),
$$

where $V:=\sum_{i=1}^{k} v_{i}$. From (2.3) we successively calculate

$$
v_{k}=\frac{1}{\varrho} \lambda^{-1} \pi_{k+1} V, \quad v_{i-1}=\varrho^{-1}\left(v_{i}+\lambda^{-1} V \pi_{i}\right), \quad i=2, \ldots, k .
$$

For $j=1, \ldots, k$ we obtain

$$
v_{j}=\lambda^{-1} V\left(\frac{1}{\varrho^{k+1-j}} \pi_{k+1}+\frac{1}{\varrho^{k-j}} \pi_{k}+\cdots+\frac{1}{\varrho} \pi_{j+1}\right) .
$$

Adding up (2.4) yields

$$
\sum_{i=1}^{k} v_{i}=V=\lambda^{-1} V\left(\pi_{k+1} \sum_{i=1}^{k} \varrho^{-i}+\pi_{k} \sum_{i=1}^{k-1} \varrho^{-i}+\cdots+\pi_{2} \varrho^{-1}\right) .
$$

Hence we obtain

$$
\varrho^{k}=\lambda^{-1}\left(\widetilde{\pi}_{2} \varrho^{k-1}+\widetilde{\pi}_{3} \varrho^{k-2}+\cdots+\widetilde{\pi}_{k} \varrho+\pi_{k+1}\right),
$$


where $\widetilde{\pi}_{n}:=\sum_{j=n}^{k+1} \pi_{j}$ for $n=2, \ldots, k+1$. We set

$$
f(\varrho):=\lambda^{-1}\left(\widetilde{\pi}_{2} \varrho^{-1}+\widetilde{\pi}_{3} \varrho^{-2}+\cdots+\widetilde{\pi}_{k} \varrho^{-(k-1)}+\pi_{k+1} \varrho^{-k}\right) .
$$

Then the equation (2.5) takes the form $f(\varrho)=1$. We see that $f(1)=r$. The function $f$ is strictly decreasing and continuous. Hence if $r=1$ then $f(1)=1$, that is, $\varrho=1$. If $f(1)=r<1$, then because $f(x) \rightarrow \infty$ as $x \rightarrow 0^{+}$, the Darboux theorem shows that the $\varrho$ which satisfies $f(\varrho)=1$ is in the interval $(0,1)$. Likewise, if $r>1$, then $\varrho>1$.

REMARK 2.3. If $\gamma \rightarrow 0_{+}$and $\pi_{2}, \ldots, \pi_{k+1} \rightarrow 0_{+}$, then $\varrho \rightarrow 0_{+}$.

Proof. If we put $\gamma=0$ or $\pi_{2}=\pi_{3}=\cdots=\pi_{k+1}=0$ into equation (2.5), it takes the form $\varrho^{k}=0$. Then the only solution is $\varrho=0$. By the continuous dependence of polynomial roots on the coefficients $\varrho$ is close to 0 when $\left(\gamma, \pi_{2}, \ldots, \pi_{k+1}\right) \in D$ is close to $(0, \ldots, 0) \in \mathbb{R}^{k+1}$.

Proposition 2.4. The coordinates of the vector $u$ satisfy

(a) $u_{1}<\cdots<u_{k}$

(b) if $\varrho=1$, then

$$
u_{l}=l u_{1}, \quad l=2, \ldots, k ; \quad u_{1}=\frac{2}{k(k+1)} .
$$

Proof. The equation $\mathcal{M} u=\varrho u$ can be written as

$$
\sum_{j=2}^{k+1} m_{j} u_{j-1}=\varrho u_{1}
$$

$$
\sum_{j=2}^{k+1} m_{j} u_{j-1}+u_{l-1}=\varrho u_{l}, \quad l=2, \ldots, k .
$$

Because $u_{j}>0, j=1, \ldots, k$, (a) is obvious.

Now, for $\varrho=1$, we add up all equations in (2.6). In view of $\sum_{j=1}^{k} u_{j}=1$ we get $k \sum_{j=1}^{k} m_{j+1} u_{j}=u_{k}$. Hence the first equation in (2.6) gives $u_{k}=k u_{1}$, and successively $u_{l}=l u_{1}, l=2, \ldots, k-1$. The proof is complete.

Definition 2.5. The subsets $D_{\mathrm{e}}, D_{\mathrm{c}}, D_{\text {sup }} \subset D$ are defined by

$$
D_{\mathrm{e}}:=\{r<1\}, \quad D_{\mathrm{c}}:=\{r=1\}, \quad D_{\text {sup }}:=\{r>1\} .
$$

It will turn out that these are the areas of parameters with ergodic, critical and supercritical chain behavior, respectively.

For the usual model of the RR processor $(k=\infty$ and there are no apparent signals) Daduna and Schassberger [2] proved the existence of an invariant probability measure when $\lambda^{-1} \mu<1$. For our model we find that for $\{\bar{Z}(n)\}$ ergodicity occurs when $\lambda^{-1} \mu<1+\lambda^{-1}$. 
Set

$$
Q(w):=E\left(\left(\sum_{j=1}^{k} w_{j}\left(\xi_{n+1, l}^{(j+1)}-m_{j+1}\right)\right)^{2}\right), \quad w \in \mathbb{R}^{k}
$$

Then

$$
Q(w)=w^{T} C w=\lambda^{-1}\left[\sum_{j=1}^{k}\left(\pi_{j+1}+\pi_{j+1}^{2}\right) w_{j}^{2}-2\left(\sum_{j=1}^{k} \pi_{j+1} w_{j}\right)^{2}\right] .
$$

We define $\mathcal{F}_{n}$ to be the $\sigma$-algebra of random events generated by $\{\bar{Z}(j)$ : $j=0,1, \ldots, n\}$. We find that

$$
E\left(\bar{\zeta}(n+1) \mid \mathcal{F}_{n}\right)=0, \quad E(\bar{\zeta}(n+1))=0, \quad n \geq 0
$$

Hence from (2.2),

$$
E(\bar{Z}(n) \cdot w)=e_{1} \cdot \sum_{i=0}^{n} \mathcal{M}^{i} w, \quad n \geq 0, w \in \mathbb{R}^{k}
$$

We also find that

$$
E((\bar{\zeta}(m) \cdot w)(\bar{\zeta}(n) \cdot w))=0, \quad m \neq n, w \in \mathbb{R}^{k}
$$

and

$$
E\left((\bar{\zeta}(n+1) \cdot w)^{2} \mid \mathcal{F}_{n}\right)=Q(w) Z(n), \quad n \geq 0, w \in \mathbb{R}^{k} .
$$

From (2.10) and (2.12) we get

$$
E\left((\bar{\zeta}(n) \cdot w)^{2}\right)=Q(w) e_{1} \sum_{i=0}^{n} \mathcal{M}^{i} \overline{1}
$$

From (2.2), (2.11), (2.13) we deduce

$$
E\left((\bar{Z}(n) \cdot w)^{2}\right)=\left(e_{1} \sum_{i=0}^{n} \mathcal{M}^{i} w\right)^{2}+\sum_{i=0}^{n-1}\left(Q\left(\mathcal{M}^{i} w\right)\left(e_{1} \sum_{j=0}^{n-i} \mathcal{M}^{j} \overline{1}\right)\right)
$$

We define

$$
X_{n}:=\bar{Z}(n) \cdot u, \quad n \geq 0
$$

By (2.1) we find that

$$
\begin{aligned}
X_{n+1} & =\varrho X_{n}+\bar{\zeta}(n+1) \cdot u+u_{1}, \quad n \geq 0, \\
X_{0} & =u_{1} .
\end{aligned}
$$

Next, from (2.10) we get

$$
E\left(X_{n}\right)=u_{1} \sum_{i=0}^{n} \varrho^{i}
$$


From (2.14) we obtain

$$
E\left(X_{n}\right)^{2}=u_{1}^{2}\left(\sum_{i=0}^{n} \varrho^{i}\right)^{2}+Q \sum_{i=0}^{n-1}\left(\varrho^{2 i}\left(e_{1} \sum_{j=0}^{n-i} \mathcal{M}^{j} \overline{1}\right)\right) .
$$

where we have set $Q \equiv Q(u)$.

\section{Main results}

3.1. Ergodicity of $\bar{Z}(n), n \in \mathbb{N}^{*}$, when $\lambda^{-1}(\mu-1)<1$

Theorem 3.1. If $r<1$ then the chain $\{\bar{Z}(n): n \in \mathbb{N}\}$ is ergodic.

3.2. Asymptotics of $\bar{Z}(n)$ as $n \rightarrow \infty$ in the critical case $\varrho=1$. We define the event $F:=\left\{\omega: \lim _{n \rightarrow \infty} Z(n)(\omega)=\infty\right\}$ and denote by $I_{F}$ its characteristic function.

Theorem 3.2. Let the parameters $\left(\gamma, \pi_{1}, \ldots, \pi_{k+1}\right)$ be such that $\varrho=1$ and $D^{2}\left(S_{l}\right)<\mu-1$. Then for every $w \in \mathbb{R}^{k}$ such that $v \cdot w>0$ the sequence

$$
\left\{\frac{1}{n} I_{F} \bar{Z}(n) \cdot w: n \in \mathbb{N}\right\}
$$

converges in distribution.

3.3. Theorem of Kesten-Stigum type when the parameters belong to $D_{\text {sup }}$. Set $Y_{n}:=\varrho^{-n} X_{n}, n \in \mathbb{N}^{*}$; here $\varrho>1$.

THEOREM 3.3. There exists a random variable $Y \in L^{2}(\Omega, P)$ such that $\lim _{n \rightarrow \infty} Y_{n}=Y$ a.e.

\section{Proofs}

4.1. Around Theorem 3.1. The main goal here is to prove the ergodicity of the chain $\{\bar{Z}(n): n \in \mathbb{N}\}$ when $r<1$. Because our chain $\{\bar{Z}(n): n \in \mathbb{N}\}$ is irreducible and homogeneous with respect to $n \in \mathbb{N}$, we can use the Foster theorem [3].

By ergodicity we mean the following property of the chain $\bar{Z}(n): n \in \mathbb{N}^{*}$ (see Theorems 1.2.3 and 1.2.4 in [3]). Set $p(y \mid x):=P(\{\bar{Z}(n+1)=y\} \mid$ $\{\bar{Z}(n)=x\}), x, y \in S$. Then there exists a unique probability measure $P_{0}$ on $S$ such that:

(i) $P_{0}(y)=\sum_{x \in S} p(y \mid x) P_{0}(x)$ for $y \in S$,

(ii) $\lim _{n \rightarrow \infty} P(\{\bar{Z}(m+n)=y\} \mid\{\bar{Z}(m)=x\})=P_{0}(y)$ for $x, y \in S$,

(iii) $P_{0}(y)>0$ for each $y \in S$.

Theorem (Foster). A homogeneous Markov chain $\left\{y_{n}: n \in \mathbb{N}\right\}$ with states in $\mathbb{Z}_{+}^{n}$ is ergodic if there exists a finite subset $A \subset \mathbb{Z}_{+}^{n}$, a function $f: \mathbb{Z}_{+}^{n} \rightarrow(0, \infty)$ and a number $\varepsilon>0$ such that 
(i) $E\left(\left(f\left(y_{k+1}\right)-f\left(y_{k}\right)\right) \mid\left\{y_{k}=x\right\}\right) \leq-\varepsilon$ for each $x \notin A$,

(ii) $E\left(f\left(y_{k+1}\right) \mid\left\{y_{k}=x\right\}\right)<\infty$ for each $x \in A$.

Proof of Theorem 3.1. We show that as $A$ in the Foster theorem we can take $A:=\{w \in S: w \cdot u \leq M\}$ with $M$ sufficiently large. For $f$ we take

$$
f(w):=\ln (3+w \cdot u), \quad w \in S .
$$

We define

$$
y_{n}:=3+\bar{Z}(n) \cdot u=3+X_{n}, \quad n \in \mathbb{N} .
$$

From the recurrence $(2.15)$ we obtain

$$
\begin{aligned}
y_{n+1} & =\varrho y_{n}+\eta_{n}+\alpha, \quad n \in \mathbb{N}^{*}, \\
y_{0} & =u_{1}+3,
\end{aligned}
$$

where $\alpha=u_{1}+3(1-\varrho)>0$ and $\eta_{n}:=\bar{\zeta}(n+1) u$ (we recall that $\varrho \in(0,1)$ ). Then $f(\bar{Z}(n)):=\ln \left(y_{n}\right)$. Now we use the following inequality (see Kersting $[5$, inequality $(2)])$ :

$$
\ln (x+h) \leq \ln x+\frac{h}{x}-\frac{1}{2}(\delta+1)^{-1} \frac{h^{2}}{x^{2}} I_{\{h \leq \delta x\}}(h),
$$

which is true for $h>-x$ and $\delta>0$. We substitute $x \equiv y_{n}$ and $h \equiv$ $(\varrho-1) y_{n}+\alpha+\eta_{n}$ into (4.1). We notice that $h=y_{n+1}-y_{n}>-y_{n}$. Then $E\left(\ln \left(y_{n+1}\right) \mid \mathcal{F}_{n}\right)$

$$
\begin{aligned}
\leq & \ln \left(y_{n}\right)+(\varrho-1)+\frac{\alpha}{y_{n}}-\frac{1}{2(1+\delta)}\left(\frac{\left(\alpha+(\varrho-1) y_{n}\right)^{2}}{y_{n}^{2}}+Q \frac{Z(n)}{y_{n}^{2}}\right) \\
& +\frac{1}{2(1+\delta)} y_{n}^{-2} E\left(\left(\alpha+(\varrho-1) y_{n}+\eta_{n}\right)^{2} I_{\left\{\alpha+(\varrho-1) y_{n}+\eta_{n} \geq \delta y_{n}\right\}} \mid \mathcal{F}_{n}\right)
\end{aligned}
$$

Let $M_{1} \geq 0$ be such that $\alpha+(\varrho-1) y \leq 0$ for $y \geq M_{1}$. If $y_{n} \geq M_{1}$, then

$$
\begin{aligned}
& E\left(\left(\alpha+(\varrho-1) y_{n}+\eta_{n}\right)^{2} I_{\left\{\alpha+(\varrho-1) y_{n}+\eta_{n} \geq \delta y_{n}\right\}} \mid \mathcal{F}_{n}\right) \\
& \quad \leq\left(\alpha+(\varrho-1) y_{n}\right)^{2} P\left(\left\{\eta_{n} \geq \delta y_{n}\right\} \mid \mathcal{F}_{n}\right)+E\left(\eta_{n}^{2} I_{\left\{\eta_{n} \geq \delta y_{n}\right\}} \mid \mathcal{F}_{n}\right)
\end{aligned}
$$

By Chebyshev's inequality,

$$
P\left(\left\{\eta_{n} \geq \delta y_{n}\right\} \mid \mathcal{F}_{n}\right) \leq \frac{1}{\delta^{2} y_{n}^{2}} E\left(\eta_{n}^{2} \mid \mathcal{F}_{n}\right)=\frac{Q Z(n)}{\delta^{2} y_{n}^{2}} \leq \frac{u_{1}^{-1} Q}{\delta^{2} y_{n}} .
$$

It follows from Hölder's and Chebyshev's inequalities that

$$
E\left(\eta_{n}^{2} I_{\left\{\eta_{n} \geq \delta y_{n}\right\}} \mid \mathcal{F}_{n}\right) \leq \frac{1}{\delta y_{n}} E\left(\left|\eta_{n}\right|^{3} \mid \mathcal{F}_{n}\right) \leq \frac{C y_{n}^{3 / 2}}{\delta y_{n}}=\frac{C}{\delta} y_{n}^{1 / 2} .
$$

Now, for $y_{n} \geq M_{1}$,

$$
\begin{aligned}
E\left(\ln \left(y_{n+1}\right) \mid \mathcal{F}_{n}\right) \leq & \ln y_{n}+\varrho-1+\alpha y_{n}^{-1} \\
& +\frac{Q u_{1}^{-1}}{2 \delta^{2}(1+\delta)}\left((1-\varrho)^{2}+\left(\frac{\alpha}{M_{1}}\right)^{2}\right) y_{n}^{-1}+\frac{C}{2 \delta(1+\delta)} y_{n}^{-3 / 2}
\end{aligned}
$$


Choose $0<\varepsilon<1-\varrho$ and $M \geq M_{1}$ such that

$$
\alpha y^{-1}+\frac{Q u_{1}^{-1}\left[(1-\varrho)^{2}+\alpha^{2} M_{1}^{-2}\right]}{2 \delta^{2}(1+\delta)} y^{-1}+\frac{C}{2 \delta(1+\delta)} y^{-3 / 2}<1-\varrho-\varepsilon
$$

for all $y \geq M$. Then, if $y_{n} \geq M$, we obtain the estimate

$$
E\left(\ln \left(y_{n+1}\right)-\ln \left(y_{n}\right) \mid \mathcal{F}_{n}\right) \leq-\varepsilon .
$$

In this way we have proved that condition (i) in the Foster theorem is satisfied. Next

$$
E\left(\ln \left(y_{n+1}\right) \mid \mathcal{F}_{n}\right) \leq E\left(2+X_{n+1} \mid \mathcal{F}_{n}\right)=2+\alpha+\varrho X_{n}=2+\alpha-3 \varrho+\varrho y_{n} .
$$

Hence condition (ii) in the Foster theorem is also satisfied. The result follows.

4.2. Around Theorem 3.2. We know that $\varrho=1$ if and only if

$$
\lambda^{-1}(\mu-1)=1 \text {. }
$$

Set

$$
d^{2}:=D^{2}\left(S_{l}\right)=\sum_{j=1}^{k+1} j^{2} \pi_{j}-\mu^{2} .
$$

Proposition 4.1. If $\varrho=1$, then $Q<2 \lambda^{-1} u_{1}^{2} d^{2}$.

Proof. We substitute $u_{l}=l u_{1}, l=1, \ldots, k$ (see Proposition 2.4) into the formula describing $Q$ :

$$
\begin{aligned}
Q & =\frac{\gamma}{1-\gamma} u_{1}^{2}\left(\sum_{j=1}^{k} j^{2}\left(\pi_{j+1}+\pi_{j+1}^{2}\right)-2\left(\sum_{j=1}^{k} j \pi_{j+1}\right)^{2}\right) \\
& <2 \frac{\gamma}{1-\gamma} u_{1}^{2}\left(\sum_{j=1}^{k} j^{2} \pi_{j+1}-\left(\sum_{j=1}^{k} j \pi_{j+1}\right)^{2}\right)=2 \frac{\gamma}{1-\gamma} u_{1}^{2} d^{2} .
\end{aligned}
$$

Proposition 4.2. There exists a nonempty subset $A \subset D$ such that $\varrho=1$ and $d^{2}<\mu-1$.

Proof. The inequality $d^{2}<\mu-1$ is satisfied when $\pi_{k+1}^{0}=1, \pi_{j}^{0}=0, j=$ $1, \ldots, k$. Then $\mu=k+1$ and in view of (4.2) with $\gamma^{0}=1 /(k+1)$ the equality $\varrho=1$ holds. By continuity $A$ also contains a nonempty neighbourhood of the point $\left(\gamma^{0}, \pi_{1}^{0}, \ldots, \pi_{k+1}^{0}\right)$.

Proposition 4.3. If $\varrho=1$ and $d^{2}<\mu-1$ then $P\left(\left\{\lim _{n \rightarrow \infty} Z(n)=\infty\right\}\right)$ $>0$ and for some $c>0$,

$$
P\left(\left\{\lim _{n \rightarrow \infty} Z(n)=\infty \text { or } \limsup _{n \rightarrow \infty} Z(n) \leq c\right\}\right)=1 .
$$

Proof. We prove the equivalent fact that $P\left(\left\{\lim _{n \rightarrow \infty} X_{n}=\infty\right\}\right)>0$ and that for some $c>0, P\left(\left\{\lim _{n \rightarrow \infty} X_{n}=\infty\right.\right.$ or $\left.\left.\limsup _{n \rightarrow \infty} X_{n} \leq c\right\}\right)=1$. 
We have a recurrent equality (see (2.15))

$$
\begin{aligned}
X_{n+1} & =X_{n}+\eta_{n}+u_{1}, \quad n \geq 0, \\
X_{0} & =u_{1},
\end{aligned}
$$

where $\eta_{n}:=\bar{\zeta}(n+1) u$. Without losing generality we assume that $X_{n} \geq 3$ for $n \in \mathbb{N}$, otherwise we consider $X_{n}+3$ instead of $X_{n}$. Thus, with $f(x):=$ $(\ln x)^{-1}$ we have $0 \leq f\left(X_{n}\right) \leq 1$.

We use the following estimate (see $[5,(6)]$ ): there exists a constant $C>0$ such that

$$
f(x+h) \leq f(x)+f^{\prime}(x) h+\frac{1}{2} f^{\prime \prime}(x) h^{2}+C \frac{|h|^{3}}{x^{3}(\ln x)^{2}}+I_{\left\{h \leq-\frac{1}{2} x\right\}}(h)
$$

for all $x \geq 3$ and $h \geq 3-x$.

After inserting

$$
f^{\prime}(x)=-\frac{1}{x(\ln x)^{2}}, \quad f^{\prime \prime}(x)=\frac{1}{x^{2}(\ln x)^{2}}+\frac{2}{x^{2}(\ln x)^{3}}
$$

we derive from (4.3) for $x \geq 3$ and $h \geq 3-x$ that

$$
\begin{aligned}
& x(\ln x)^{2} {[f(x)-f(x+h)] } \\
& \geq h-\frac{1}{2}\left[\frac{1}{x}+\frac{2}{x \ln x}\right] h^{2}-C \frac{|h|^{3}}{x^{2}}-x(\ln x)^{2} I_{\left\{h \leq-\frac{1}{2} x\right\}}(h) .
\end{aligned}
$$

In (4.4) we now substitute $X_{n}$ for $x$ and $X_{n+1}-X_{n}$ for $h$. Because $X_{n+1}-$ $X_{n}=u_{1}+\eta_{n}$, we derive

$$
\begin{aligned}
& X_{n}\left(\ln X_{n}\right)^{2} E\left[\left(f\left(X_{n}\right)-f\left(X_{n+1}\right)\right) \mid \mathcal{F}_{n}\right] \\
& \geq u_{1}-\frac{1}{2}\left(\frac{1}{X_{n}}+\frac{2}{X_{n} \ln X_{n}}\right)\left(Q u_{1}^{-1} X_{n}+u_{1}^{2}\right) \\
& \quad-C_{1} X_{n}^{-1 / 2}-X_{n}\left(\ln X_{n}\right)^{2} P\left(\left\{X_{n+1}-X_{n} \leq-X_{n} / 2\right\} \mid \mathcal{F}_{n}\right) .
\end{aligned}
$$

From Chebyshev's inequality we obtain

$$
P\left(\left\{X_{n+1}-X_{n} \leq-X_{n} / 2\right\} \mid \mathcal{F}_{n}\right) \leq 8 X_{n}^{-3} E\left(\left|X_{n+1}-X_{n}\right|^{3} \mid \mathcal{F}_{n}\right) .
$$

Now we have

$$
\begin{aligned}
E\left(\left|X_{n+1}-X_{n}\right|^{3} \mid \mathcal{F}_{n}\right) & =E\left(\left|u_{1}+\eta_{n}\right|^{3} \mid \mathcal{F}_{n}\right) \\
& \leq u_{1}^{3}+3 u_{1}^{2} E\left(\left|\eta_{n}\right| \mid \mathcal{F}_{n}\right)+3 u_{1} E\left(\eta_{n}^{2} \mid \mathcal{F}_{n}\right)+E\left(\left|\eta_{n}\right|^{3} \mid \mathcal{F}_{n}\right) \\
& \leq u_{1}^{3}+3 u_{1}^{2}(Q Z(n))^{1 / 2}+3 u_{1} Q Z(n)+E\left(\left|\eta_{n}\right|^{3} \mid \mathcal{F}_{n}\right) .
\end{aligned}
$$

In deriving the last inequality we have taken into account first $E\left(\left|\eta_{n}\right| \mid \mathcal{F}_{n}\right) \leq$ $\left(E\left(\eta_{n}^{2} \mid \mathcal{F}_{n}\right)\right)^{1 / 2}$ and then $(2.12)$ with $Q \equiv Q(u)$.

From the Cauchy inequality we estimate

$$
\begin{aligned}
E\left(\left|\eta_{n}\right|^{3} \mid \mathcal{F}_{n}\right) & =E\left(\eta_{n}^{2}\left|\eta_{n}\right| \mid \mathcal{F}_{n}\right) \leq \sqrt{E\left(\eta_{n}^{4} \mid \mathcal{F}_{n}\right)} \cdot \sqrt{E\left(\eta_{n}^{2} \mid \mathcal{F}_{n}\right)} \\
& =\sqrt{E\left(\eta_{n}^{4} \mid \mathcal{F}_{n}\right)} \cdot \sqrt{Q Z(n)} .
\end{aligned}
$$


We write

$$
\begin{aligned}
\eta_{n}^{4} & =\left(\sum_{j=1}^{k} u_{j} \zeta_{j+1}(n+1)\right)^{4} \\
= & \sum_{l_{1}, l_{2}, l_{3}, l_{4}=1}^{k} u_{l_{1}} \zeta_{l_{1}+1}(n+1) \cdot u_{l_{2}} \zeta_{l_{2}+1}(n+1) \\
& \times u_{l_{3}} \zeta_{l_{3}+1}(n+1) \cdot u_{l_{4}} \zeta_{l_{4}+1}(n+1) \\
\leq & \sum_{l_{1}, l_{2}, l_{3}, l_{4}=1}^{k} \sum_{m=1}^{4}\left(u_{l_{m}} \zeta_{l_{m}+1}(n+1)\right)^{4}=k^{4} \sum_{j=1}^{k}\left(u_{j} \zeta_{j+1}(n+1)\right)^{4} .
\end{aligned}
$$

Next, we write

$$
\begin{aligned}
\left(\zeta_{j+1}(n+1)\right)^{4} & =\left(\sum_{l=1}^{Z(n)} \dot{\circ}_{n, l}^{\circ}(j+1)\right)^{4} \\
& =\sum_{l=1}^{Z(n)}\left(\dot{\xi}_{n, l}^{(j+1)}\right)^{4}+\sum_{l_{1}, l_{2}=1, l_{1} \neq l_{2}}^{Z(n)}\left(\dot{\circ}_{n, l_{1}}^{(j+1)}\right)^{2}\left(\dot{\xi}_{n, l_{2}}^{(j+1)}\right)^{2}+r
\end{aligned}
$$

where $\stackrel{\circ}{\xi}_{n, l}^{(j)}:=\xi_{n, l}^{(j)}-m_{j}$ and $E\left(r \mid \mathcal{F}_{n}\right)=0$ because $\left\{\stackrel{\circ}{\xi}_{n, 1}^{(j)}, \ldots, \dot{\xi}_{n, Z(n)}^{(j)}\right\}$ are independent. Collecting these together we obtain

$$
E\left(\eta_{n}^{4} \mid \mathcal{F}_{n}\right) \leq k^{4} \sum_{j=1}^{k} u_{j}^{4}\left[C_{j+1}^{(4)} Z(n)+\left(C_{j+1}^{(2)}\right)^{2}(Z(n))^{2}\right]=c_{1} Z(n)+c_{2}(Z(n))^{2}
$$

where $C_{j}^{(2)}:=E\left(\left(\dot{\xi}_{n, l}^{(j)}\right)^{2}\right)$ and $C_{j}^{(4)}:=E\left(\left(\dot{\xi}_{n, l}^{(j)}\right)^{4}\right)$ are independent of $n, l$ (see Section 1).

Notice that $u_{1}^{-1} X_{n} \geq Z(n)$. Thus, coming back to (4.6), we have proved

$$
\begin{aligned}
P\left(\left\{X_{n+1}-\right.\right. & \left.\left.X_{n} \leq-X_{n} / 2\right\} \mid \mathcal{F}_{n}\right) \\
\leq & 8 X_{n}^{-3}\left[u_{1}^{3}+3 u_{1}^{3 / 2} Q^{1 / 2} X_{n}^{1 / 2}+3 Q X_{n}\right. \\
& \left.+Q^{1 / 2} u_{1}^{-1 / 2} X_{n}^{1 / 2}\left(c_{1} u_{1}^{-1} X_{n}+c_{2} u_{1}^{-2} X_{n}^{2}\right)^{1 / 2}\right] \\
\leq & 8\left[u_{1}^{3} X_{n}^{-3}+3 u_{1}^{3 / 2} Q^{1 / 2} X_{n}^{-5 / 2}\right. \\
& \left.+\left(3 Q+Q^{1 / 2} u_{1}^{-1} c_{1}^{1 / 2}\right) X_{n}^{-2}+c_{2}^{1 / 2} Q^{1 / 2} u_{1}^{-3 / 2} X_{n}^{-3 / 2}\right] .
\end{aligned}
$$

In view of Propositions 4.1 and 4.2 we get

$$
\frac{Q}{2} u_{1}^{-1}<u_{1}
$$

From this and (4.7) we conclude that there exists $M>0$ such that the right side in (4.5) is positive when $X_{n} \geq M$. Taking into account the form of the 
left side in (4.5) we derive

$$
f\left(X_{n}\right) \geq E\left(f\left(X_{n+1}\right) \mid \mathcal{F}_{n}\right) \quad \text { if } X_{n} \geq M .
$$

This in turn leads to the conclusion that the sequence

$$
W_{n}:=\min \left\{f\left(X_{n}\right), f(M)\right\}, \quad n \in \mathbb{N},
$$

is a nonnegative supermartingale such that $E\left(W_{n}\right) \leq 1, n \in \mathbb{N}$. Thus, the sequence $\left\{W_{n}: n \in \mathbb{N}\right\}$ is convergent almost everywhere and the expected values $\left\{E\left(W_{n}\right): n \in \mathbb{N}\right\}$ are also convergent.

Further the proof runs exactly in the same way as the end of the proof of Theorem 2 in [5]. This completes the proof of Proposition 4.3.

Proposition 4.4. If $\varrho=1$, then

$$
\begin{aligned}
\lim _{n \rightarrow \infty} n^{-1} E(\bar{Z}(n) \cdot w) & =u_{1}(v \cdot w), \\
\lim _{n \rightarrow \infty} n^{-2} E\left((\bar{Z}(n) \cdot w)^{2}\right) & =u_{1}(v \cdot w)^{2}\left(u_{1}+\frac{Q}{2} \sum_{i=1}^{k} v_{i}\right),
\end{aligned}
$$

where $w \in \mathbb{R}^{k}$ is a column vector.

Proof. We know (see e.g. [7, Theorem 6.1, p. 14]) that $\mathcal{M}^{l}=\varrho^{l} u v+\mathcal{M}_{1}^{(l)}$, where $\left\|\mathcal{M}_{1}^{(l)}\right\| \leq C \alpha^{l}$ with $\alpha$ independent of $l$, and $0<\alpha<\varrho$. Then for $\varrho=1$ we obtain directly from $(2.10)$

$$
\lim _{n \rightarrow \infty} n^{-1} E(\bar{Z}(n) \cdot w)=u_{1}(v \cdot w) .
$$

Using (2.10) and (2.14) we obtain

$$
\begin{gathered}
E\left((\bar{Z}(n+1) \cdot w)^{2}\right)=\left(e_{1} \sum_{l=0}^{n+1} \mathcal{M}^{l} w\right)^{2}+\sum_{l=0}^{n} E\left(\left(\bar{\zeta}(n+1-l) \mathcal{M}^{l} w\right)^{2}\right), \\
E\left(\left(\bar{\zeta}(n+1-l) \mathcal{M}^{l} w\right)^{2}\right)=Q\left(\mathcal{M}^{l} w\right) E(Z(n+1-l)) .
\end{gathered}
$$

For $\varrho=1$ we have

$$
Q\left(\mathcal{M}^{l} w\right)=(v \cdot w)^{2} Q+2(v \cdot w)\left(u_{T} \mathcal{C M}_{1}^{(l)} w\right)+Q\left(\mathcal{M}_{1}^{(l)} w\right) .
$$

This yields

$$
\begin{aligned}
\lim _{n \rightarrow \infty} & n^{-2} \sum_{l=0}^{n} E\left(\left(\bar{\zeta}(n+1-l) \mathcal{M}^{l} w\right)^{2}\right) \\
& =(v \cdot w)^{2} Q \lim _{n \rightarrow \infty} n^{-2} \sum_{l=0}^{n} E(Z(n+1-l))=(v \cdot w)^{2} Q \frac{u_{1}}{2} \sum_{j=1}^{n} v_{j} .
\end{aligned}
$$

Here we have used the fact that

The proof is complete.

$$
\lim _{n \rightarrow \infty} n^{-1} E(Z(n))=u_{1} \sum_{j=1}^{k} v_{j} .
$$


Before we derive Theorem 3.2, we recall the following auxiliary lemma from $[6]$.

Lemma 4.5. Let the sequences $\left\{a_{l}: l \in \mathbb{N}\right\},\left\{b_{l} ; l \in \mathbb{N}\right\} \subset(0, \infty)$ satisfy:

$$
\sum_{i=1}^{n} a_{i}=A(n) \nearrow \infty, \quad \lim _{n \rightarrow \infty} \frac{A(n-m)}{A(n)}=1 \quad \text { for each } m \in \mathbb{N},
$$

and

$$
\lim _{n \rightarrow \infty} b_{n}=b .
$$

Then

$$
\left|\sum_{i=1}^{n} a_{i} b_{i}-b \sum_{i=1}^{n} a_{i}\right|=o\left(\sum_{i=1}^{n} a_{i}\right) .
$$

Proof of Theorem 3.2. Let $w \in \mathbb{R}^{k}$ (column vector) be as in the statement of the theorem. We will prove that for all such $w$ and all $m \in \mathbb{N}$ the limit

$$
\lim _{n \rightarrow \infty} n^{-m} E\left(I_{F}(\bar{Z}(n) \cdot w)^{m}\right)=: \mu(w, m)
$$

exists. From the recurrence (2.1) we obtain

$$
\bar{Z}(n+1) w=\bar{Z}(n) \mathcal{M} w+\eta_{n}(w)+w_{1}
$$

where $\eta_{n}(w):=\bar{\zeta}(n+1) \cdot w$. We know that the limits $\mu(w, 1), \mu(w, 2)$ exist (see Propositions 4.3 and 4.4). Now we use induction on $j$. Assume that the limits $\mu(w, j), 1 \leq j \leq m-1, m \geq 3$ exist. We will prove the existence of $\mu(w, m)$.

From (4.8) we derive the expansion

where

$$
\begin{aligned}
E\left((\bar{Z}(n+1) w)^{m} \mid\right. & \left.\mathcal{F}_{n}\right)=\left(w_{1}+\bar{Z}(n) \mathcal{M} w\right)^{m} \\
& +Q(w)\left(\begin{array}{c}
m \\
2
\end{array}\right)\left(w_{1}+\bar{Z}(n) \mathcal{M} w\right)^{m-2} Z(n)+r_{n, m}(w)
\end{aligned}
$$

$$
r_{n, m}(w):=\sum_{j=0}^{m-3}\left(\begin{array}{c}
m \\
j
\end{array}\right)\left(w_{1}+\bar{Z}(n) \mathcal{M} w\right)^{j} E\left(\eta_{n}(w)^{m-j} \mid \mathcal{F}_{n}\right) .
$$

Now we insert into (4.9):

$$
\begin{aligned}
\left(w_{1}+\bar{Z}(n) \mathcal{M} w\right)^{m} & =(\bar{Z}(n) \mathcal{M} w)^{m}+m w_{1}(\bar{Z}(n) \mathcal{M} w)^{m-1}+r 1_{n, m}(w), \\
\left(w_{1}+\bar{Z}(n) \mathcal{M} w\right)^{m-2} & =(\bar{Z}(n) \mathcal{M} w)^{m-2}+r 2_{n, m}(w),
\end{aligned}
$$

where

$$
\begin{aligned}
& r 1_{n, m}(w):=\sum_{j=0}^{m-2}\left(\begin{array}{c}
m \\
j
\end{array}\right)(\bar{Z}(n) \mathcal{M} w)^{j} w_{1}^{m-j} \\
& r 2_{n, m}(w):=\sum_{j=0}^{m-3}\left(\begin{array}{c}
m-2 \\
j
\end{array}\right)(\bar{Z}(n) \mathcal{M} w)^{j} w_{1}^{m-2-j}
\end{aligned}
$$


We obtain

$$
\begin{aligned}
E\left((\bar{Z}(n+1) w)^{m}\right) & \\
= & E\left((\bar{Z}(n) \mathcal{M} w)^{m}\right)+m w_{1} E\left((\bar{Z}(n) \mathcal{M} w)^{m-1}\right) \\
& +Q(w)\left(\begin{array}{c}
m \\
2
\end{array}\right) E\left[(\bar{Z}(n) \mathcal{M} w)^{m-2} Z(n)\right] \\
& +E\left[r_{n, m}(w)+r 1_{n, m}(w)+Q(w)\left(\begin{array}{c}
m \\
2
\end{array}\right) Z(n) r 2_{n, m}(w)\right] .
\end{aligned}
$$

Denote the last expectation by $\varrho_{n, m}(w)$. Iterating $(4.10)$ with respect to $n$ gives

$$
\begin{aligned}
& E\left((\bar{Z}(n+1) w)^{m}\right) \\
= & \left.E\left(\bar{Z}(1) \mathcal{M}^{n} w\right)^{m}\right)+m \cdot \sum_{l=1}^{n}\left(\mathcal{M}^{l-1} w\right)_{1} E\left(\left(\bar{Z}(n+1-l) \mathcal{M}^{l} w\right)^{m-1}\right) \\
& +\left(\begin{array}{c}
m \\
2
\end{array}\right) \sum_{l=1}^{n} Q\left(\mathcal{M}^{l-1} w\right) E\left(\left(I_{F} \bar{Z}(n+1-l) \mathcal{M}^{l} w\right)^{m-2} Z(n+1-l)\right) \\
& +\sum_{l=1}^{n} \varrho_{n-l, m}\left(\mathcal{M}^{l-1} w\right) .
\end{aligned}
$$

We find directly that

$$
\varrho_{n-l, m}\left(\mathcal{M}^{l-1} w\right)=o\left(E\left(\left(I_{F} Z(n)\right)^{m-1}\right)\right) \quad \text { as } I_{F} Z(n) \rightarrow \infty,
$$

which leads to

$$
n^{-m} \sum_{l=1}^{n} \varrho_{n-l, m}\left(\mathcal{M}^{l-1} w\right) \rightarrow 0 \quad \text { as } n \rightarrow \infty
$$

Next, analogously to $[6,(1.18),(1.19)]$, basing on Lemma 4.5 we find that

$$
\begin{aligned}
\sum_{l=1}^{n}\left(\mathcal{M}^{l-1} w\right)_{1} E\left(\left(\bar{Z}(n+1-l) \mathcal{M}^{l} w\right)^{m-1}\right) & \\
& =\left(e_{1} s\right) \sum_{l=1}^{n} E(\bar{Z}(l) s)^{m-1}+o\left(n^{m}\right)
\end{aligned}
$$

and

$$
\begin{aligned}
\sum_{l=1}^{n} Q\left(\mathcal{M}^{l-1} w\right) E((\bar{Z}(n & \left.\left.+1-l) \mathcal{M}^{l} w\right)^{m-2} Z(n+1-l)\right) \\
& =Q(s) \sum_{l=1}^{n} E\left((\bar{Z}(l) s)^{m-2} Z(l)\right)+o\left(n^{m}\right),
\end{aligned}
$$

where $s:=(v \cdot w) u$. For details see [6]. 
Using now the induction assumption we obtain

$$
n^{-m} \sum_{l=1}^{n} E\left(I_{F}(\bar{Z}(l) s)^{m-1}\right) \rightarrow \mu(s, m-1) \quad \text { as } n \rightarrow \infty .
$$

Using the induction assumption once more, for $0<r \leq m-1$, we infer that

$$
\lim _{n \rightarrow \infty} n^{-r} E\left((\bar{Z}(n))^{l}\right)=a(l, r)
$$

where $(\bar{Z})^{l}=Z_{1}^{l_{1}} \cdots Z_{k}^{l_{k}}, \sum_{j=1}^{n} l_{j}=r, l_{j} \geq 0, j=1, \ldots, k$. For details see the proof of Theorem 1 in [6].

We can write

$$
\mu(w, r)=\sum_{\left\{l: l_{j} \geq 0, \sum_{j=1}^{k} l_{j}=r\right\}} \overline{\left(\begin{array}{l}
r \\
l
\end{array}\right)} a(l, r) w^{l}, \quad 0<r \leq m-1,
$$

where $w^{l}:=w_{1}^{l_{1}} \cdots w_{k}^{l_{k}}$. From this we derive

$$
\begin{aligned}
& \lim _{n \rightarrow \infty} n^{-(m-1)} E\left(I_{F}(\bar{Z}(n) s)^{m-2} Z(n)\right) \\
= & u_{1}-(v \cdot w)^{m-2} \sum_{i=1}^{k} \sum_{\left\{l: l_{j} \geq 0, \sum_{j=1}^{k} l_{j}=m-2\right\}} \overline{\left(\begin{array}{c}
k-2 \\
l
\end{array}\right)} a\left(l+e_{i}, m-1\right) u^{l} .
\end{aligned}
$$

Now by (4.13), (4.14), (4.16) we deduce from (4.11) that

$$
\begin{aligned}
\mu(w, m) \equiv & \lim _{n \rightarrow \infty} n^{-m} E\left(I_{F}(\bar{Z}(n+1) w)^{m}\right) \\
= & u_{1}(v \cdot w) \mu(s, m-1)+(v \cdot w)^{m} Q \frac{m-1}{2} \\
& \times \sum_{i=1}^{k} \sum_{\left\{l: l_{j} \geq 0, \sum_{j=1}^{k} l_{j}=m-2\right\}} \overline{\left(\begin{array}{c}
m-2 \\
l
\end{array}\right)} a\left(l+e_{i}, m-1\right) u^{l} \\
= & (v \cdot w)^{m} u_{1} \mu(u, m-1)+(v \cdot w)^{m} \frac{Q(m-1)}{2} \sum_{i=1}^{k} u_{i}^{-1} \\
& \times \sum_{\left\{l: l_{j} \geq 0, \sum_{j=1}^{k} l_{j}=m-2\right\}} \overline{\left(\begin{array}{c}
m-2 \\
l
\end{array}\right)} a\left(l+e_{i}, m-1\right) u^{l} u_{i} .
\end{aligned}
$$

Because $v \cdot u=1$, from (4.17) we obtain

$$
\mu(u, m) \leq\left(u_{1}+Q u_{1}^{-1} \frac{m-1}{2}\right) \mu(u, m-1) .
$$

Thus

$$
\mu(u, m) \leq\left(\frac{Q u_{1}^{-1}}{2}\right)^{m} \Gamma\left(\frac{2 u_{1}^{2}}{Q}+m\right) \cdot \frac{2 u_{1}^{2}}{Q \Gamma\left(2 u_{1}^{2} / Q\right)}
$$


This estimate shows that the series

$$
\sum_{m=0}^{\infty}(-1)^{m} \frac{\mu(u, m)}{m !} t^{m}
$$

is convergent on some $t$-interval containing 0 .

Thus the sequence of moments $\{\mu(u, m): m \in \mathbb{N}\}$ defines exactly a certain probability distribution. Since (4.17) yields $\mu(w, m)=(v \cdot w)^{m} \mu(u, m)$, $m \in \mathbb{N}$, also the sequence $\{\mu(w, m): m \in \mathbb{N}\}$ defines a probability distribution. In particular, if $w=\overline{1}$ then the sequence $\left\{n^{-1} I_{F} Z(n): n \in \mathbb{N}\right\}$ is convergent in distribution and the limiting distribution has moments $\{\mu(\overline{1}, m): m \in \mathbb{N}\}$. The proof is complete.

Let $Z$ denote the random variable with distribution

We note that

$$
\lim _{n \rightarrow \infty} P\left(\left\{I_{F} \frac{1}{n} Z(n)<x\right\}\right), \quad x \in \mathbb{R} .
$$

which yields

$$
D^{2}(Z)=\frac{Q}{2} u_{1}\left(\sum_{j=1}^{k} v_{j}\right)^{3}>0
$$

REMARK 4.6. The random variable $Z$ is not constant a.e.

4.3. Proof of Theorem 3.3. Everywhere in this section $\varrho>1$. From (2.15) we get

$$
\begin{aligned}
Y_{n+1} & =Y_{n}+\varrho^{-(n+1)} \eta_{n}+\varrho^{-(n+1)} u_{1}, \quad n \geq 0, \\
Y_{0} & =u_{1} .
\end{aligned}
$$

It is not difficult to obtain

$$
\lim _{n \rightarrow \infty} E\left(Y_{n}\right)=u_{1} \frac{\varrho}{\varrho-1} .
$$

We use the scheme of proof from [11]. Set $\Delta Y_{n}:=Y_{n+1}-Y_{n}, n \in \mathbb{N}^{*}$. We calculate

and

$$
E\left(\Delta Y_{i} \Delta Y_{j}\right)=\varrho^{-(i+j+2)} u_{1}^{2}, \quad i \neq j
$$

$$
\begin{aligned}
E\left(\left(\Delta Y_{i}\right)^{2}\right) & =\varrho^{-2(i+1)}\left(u_{1}^{2}+Q E(Z(i))\right) \\
& \leq \varrho^{-2(i+1)} u_{1}^{2}+Q u_{1}^{-1} \varrho^{-2(i+1)} E\left(Y_{i}\right) \\
& =\varrho^{-2(i+1)}\left(u_{1}^{2}+Q \frac{\varrho^{i+1}-1}{\varrho-1}\right)<C \varrho^{-(i+1)},
\end{aligned}
$$

where $C>0$ is a constant. Now we can estimate

$$
\text { (4.21) } E\left(\left(Y_{n+m}-Y_{n}\right)^{2}\right)=\sum_{i=n}^{n+m-1} E\left(\left(\Delta Y_{i}\right)^{2}\right)+2 \sum_{i, j=n, i \neq j}^{n+m-1} E\left(\Delta Y_{i} \Delta Y_{j}\right)
$$




$$
<C \sum_{i=n}^{n+m-1} \varrho^{-(i+1)}+2 u_{1}^{2}\left(\sum_{i=n}^{n+m-1} \varrho^{-(i+1)}\right)^{2} .
$$

Therefore $\lim _{n \rightarrow \infty} E\left(\left(Y_{n+m}-Y_{n}\right)^{2}\right)=0$, uniformly in $m \in \mathbb{N}$. Hence there exists $Y \in L^{2}(\Omega, P)$ such that $\lim _{n \rightarrow \infty} E\left(\left(Y_{n}-Y\right)^{2}\right)=0$. If we let $m \rightarrow \infty$ on both sides of (4.21) we get

$$
E\left(\left(Y_{n}-Y\right)^{2}\right)<C_{1} \varrho^{-(n+1)}, \quad n \in \mathbb{N} .
$$

Hence the series $\sum_{n=1}^{\infty} E\left(\left(Y_{n}-Y\right)^{2}\right)$ converges. This gives $P\left(\left\{\lim _{n \rightarrow \infty} Y_{n}\right.\right.$ $=Y\})=1$. The proof is finished.

\section{References}

[1] H. Daduna, Queueing Networks with Discrete Time Scale, Lecture Notes in Comput. Sci. 2046, Springer, 2001.

[2] H. Daduna and R. Schassberger, A discrete-time round-robin queue with Bernoulli input and general arithmetic service time distribution, Acta Inform. 15 (1981), 251263.

[3] G. Fayolle, V. A. Malyshev and M. V. Men'shikov, Topics in Constructive Theory of Countable Markov Chains, Cambridge Univ. Press, 1995.

[4] S. A. Grishechkin, One-channel systems with round robin or processor sharing, and branching processes, Mat. Zametki 44 (1988), 433-448 (in Russian).

[5] G. Kersting, On recurrence and transience of growth models, J. Appl. Probab. 23 (1986), 614-625.

[6] F. C. Klebaner, Linear growth in near-critical population-size-dependent multitype Galton-Watson processes, ibid. 26 (1989), 431-445.

[7] J. C. Mode, Multitype Branching Processes. Theory and Applications, American Elsevier, New York, 1971.

[8] S. Sagitov, Linear growth in the multi-type Galton-Watson process with density dependent reproduction", Chalmers Univ. of Technology, Math. Dept., Preprint no. 30 (1998), 24 pp.

[9] R. Schassberger, On the response time distribution in a round-robin queue, Acta Inform. 16 (1981), 57-62.

[10] - A new approach to the $M / G / 1$ processor-sharing queue, Adv. Appl. Probab. 16 (1984), 202-213.

[11] B. A. Sevast'yanov, Branching Processes, Nauka, Moscow, 1971 (in Russian).

Institute of Mathematics and Physics

University of Technology and Agriculture

S. Kaliskiego 7

85-796 Bydgoszcz, Poland

E-mail: anel@mail.atr.bydgoszcz.pl

Received on 4.3.2005;

revised version on 13.4 .2006 